\title{
Strong Commutativity of Unbounded Self-adjoint Operators on a Separable Hilbert space
}

\author{
Fidelis Mukudi $^{*} \quad$ Justus Mile $^{2} \quad$ Lucy Chikamai $^{2} \quad$ Shem Aywa $^{2}$ \\ 1.Department of Mathematics, Kibabii University, PO box 1699-50200. Bungoma, Kenya \\ 2.Department of Mathematics, Kiriri Women's University of Science and Technology, PO box $49274-00100$, \\ Nairobi, Kenya \\ 3.Department of Mathematics, Kibabii University, PO box 1699-50200. Bungoma, Kenya \\ 4.Department of Mathematics, Kibabii University, PO box 1699-50200. Bungoma, Kenya
}

\begin{abstract}
The unbounded Self-adjoint operators that strongly commute on a common dense subset of their domain commute pointwise. When the operators commute pointwise on the same dense subset, there is to guarantee that they will commute strongly. By imposing some conditions, we on the operators as well as the underlying space, we get pointwise commuting unbounded operators that commute strongly. This article shows that by suitably selecting two unbounded positive Self-adjoint operators with compact inverses we get a set of pointwise commuting self-adjoint operators that commute on common core. then prove that it strongly commutes on the same subspace.
\end{abstract}

Keywords: Unbounded operators, Self-adjoint operators, Commutative operators

DOI: $10.7176 / \mathrm{MTM} / 10-8-03$

Publication date: December $31^{\text {st }} 2020$

\section{Introduction}

The unbounded Self-adjoint operators are said to be commute strongly if their bounded transforms commute. Equivalently, the unbounded Self-adjoint operators commute strongly if their spectral measures commute [8]. It is known that strongly commuting unbonded Self-adjoint operators that commute on a common core also commute pointwise, however, the pointwise commuting unbounded Self-adjoint operators do not necessarily commute strongly. This was discovered by Nelson in [4] when he sited an example of a pair unbounded Selfadjoint operators that commute pointwise on a common core but do not commute strongly. These led to a series of studies of such operators as discussed in $[\mathbf{5 , 6 , 7 ]}$.

In general, pointwise commuting unbounded Self-adjoint operators do not commute strongly. However, with the provision of conditions on the operators as well as the underlying space, this aspect in question can be achieved. In this article, we show that by making the underlying space be a separable Hilbert Space and suitably selecting a special positive operator, the pointwise commutating unbounded Self-adjoint operators commute strongly.

\section{Preliminary Concepts}

In this section, we briefly provide the preliminary concepts of the paper. We will use $\mathbb{H}$ to denote a Complex valued Hilbert space over a field $\mathbb{F}$. We will use $E$ and $F$ to denote the two pairs of pointwise unbounded Selfadjoint operators with domains $D(E)$ and $D(F)$ and spectral measures denoted by $P_{E}(\lambda)$ and $P_{F}(\lambda)$ respectively. The range of the operate $F$ will be denoted by $\operatorname{Ran}(F)$. The restriction of an operator $E$ on $\mathbb{H}_{i} \subseteq \mathbb{H}$ will be denoted by $\left.E\right|_{\mathbb{H}_{I}}$ and the commutator of two operators $E$ and $F$ will be denoted by $[E, F]=E F-F E$. We begin by some definitions.

Definition: Separable Hilbert Space

A Hilbert space $\mathbb{H}$ is separable if it can be represented in the form

\section{Definition: Compact operator}

$$
\mathbb{H}=\bigoplus_{i \in \mathbb{N}} \mathbb{H}_{i}
$$

An operator $E: \mathbb{H}_{1} \rightarrow \mathbb{H}_{2}$ is compact if the closure of $E(C)$ that is, $\overline{E(C)} \in \mathbb{H}_{2}$ is compact for a compact set $C \in$ $\mathbb{H}_{2}$.

The results in [2] provides a way of constructing unbounded Self-adjoint operators that are pointwise commutative, hence strongly commutative. The result states in part that if A is a closed and densely defined Dirichlet operator on a square-integrable space that is associated with a uniformly elliptic differential operator, then there exists a sufficiently large $\alpha_{0}$ such that $(A+\alpha)^{-1}$ exists given that $\alpha \geq \alpha_{0} \in \mathbb{R}$.

This is a case of unbounded operators with compact inverses. In this paper, we will have $A$ and $B$ be unbounded positive Self-adjoint operators as they satisfy the hypothesis of the above results. Let $\alpha_{A 0}$ and $\alpha_{B 0}$ be 
sufficiently such that $A+\alpha_{A}$ and $B+\alpha_{B}$ are invertible for $\alpha_{A} \geq \alpha_{A 0} \in \mathbb{R}$ and $\alpha_{B} \geq \alpha_{B 0} \in \mathbb{R}$. Define $\alpha_{0}=\max \left\{\alpha_{A 0}, \alpha_{B 0}\right\}$, then $E=A+\alpha$ and $F=B+\alpha$ are invertible for $\alpha \geq \alpha_{0} \in \mathbb{R}$, thus $(A+\alpha)^{-1}$ and $(B+\alpha)^{-1}$ exists and are compact operators. Compact operators are always bounded, hence $(A+\alpha)^{-1}$ and $(B+\alpha)^{-1}$ are bounded operators.

The operators $A$ and $B$ are bounded self-adjoint operators hence, by the spectral theorem [8], there exists unique spectral measures $P_{A}(\lambda)$ and $P_{B}\left(\lambda^{*}\right)$ such that

$$
A=\int_{\mathcal{J}} \lambda d P_{A}(\lambda) \text { and } B=\int_{\mathcal{J}^{*}} \lambda^{*} d P_{B}\left(\lambda^{*}\right)
$$

for $\sigma(A) \in \mathcal{J}$ and $\sigma(B) \in \mathcal{J}^{*}$ where $\mathcal{J}$ and $\mathcal{J}^{*}$ are compact invervals on $\mathbb{R}$ and $\lambda, \lambda^{*} \in \mathbb{R}$. The basics of unbounded Self-adjoint operators and their functional calculus are found in $[\mathbf{1}, \mathbf{3}, \mathbf{8}]$. Using the functional calculus for self-adjoint operators, the spectral representation for $(A+\alpha)^{-1}$ and $(B+\alpha)^{-1}$ are

$$
\begin{aligned}
& (A+\alpha)^{-1}=\int_{\jmath} \frac{1}{\lambda+\alpha} d P_{A}(\lambda) \\
& (B+\alpha)^{-1}=\int_{\jmath} \frac{1}{\lambda^{*}+\alpha} d P_{B}\left(\lambda^{*}\right)
\end{aligned}
$$

The functions $f(\lambda)=\frac{1}{\lambda+\alpha} \in \mathbb{R}$ and $g\left(\lambda^{*}\right)=\frac{1}{\lambda^{*}+\alpha} \in \mathbb{R}$ because $\lambda, \lambda^{*}, \alpha \in \mathbb{R}$. Thus, the operators $(A+$ $\alpha)^{-1}$ and $(B+\alpha)^{-1}$ are Self-adjoint operators. Likewise, $E=A+\alpha$ and $F=A+\alpha$ are positive Self-adjoint operators. By choice, $A$ and $B$ were unbounded Self-aajoint operators, consquently, $E=A+\alpha$ and $F=A+\alpha$ are unbounded, hence, unbounded positive Self-adjoint operators.

Having established that $E$ and $F$ are unbounded positive Self-adjoint operators with compact inverses $(A+\alpha)^{-1}$ and $(B+\alpha)^{-1}$ respectively, we move forward to investigate the commutativity of these operators.

\section{Commutativity of Unbounded Self-adjoint operators}

This section provides the main results of this article. We will show that suitably selected unbounded positive Self-adjoint operators commute pointwise on a common core. We will further show this pointwise commutativity implies strong commutativity, for that selected case. We begin by providing a proposition with respect to the first statement above.

Proposition 3.1

Let $E=A+\alpha$ and $F=B+\alpha$ be unbounded positive Self-adjoint operators having compact Self-adjoint inverses on a Hilbert Space $\mathbb{H}$ for sufficiently large $\alpha \in \mathbb{R}$. Let the inverses be $T=(A+\alpha)^{-1}$ and $S=$ $(B+\alpha)^{-1}$ respectively. Define $\mathcal{C}$ as $\mathcal{C}=(A+\alpha)^{-1}(B+\alpha)^{-1}(I-P) \mathbb{H}=T S(I-P) \mathbb{H}$ where $P$ is a projection on $\overline{\left[(A+\alpha)^{-1},(B+\alpha)^{-1}\right] \mathbb{H}}=\overline{[\mathrm{TS}] \mathbb{H}}$. Then

1. $\mathcal{C} \subseteq D(E F) \cap D(F E)$ and $E F x=F E x$ for all $x \in \mathcal{C}$

2. If $P \mathbb{H} \cap T H \mathbb{H}=P \mathbb{H} \cap S \mathbb{H}=\{0\}$, then $\mathcal{C}$ is a core for $E$ and $F$

3. $F$ and $E$ are invariant under $\mathcal{C}$

\section{Proof}

Let $v \in \mathbb{H}$ be arbitrary. Since $P$ is a projection on $\overline{[\mathrm{TS}] \mathbb{H}}$, then $I-P$ is a projection on $(\overline{[\mathrm{TS}] \mathbb{H}})^{\perp}$. As such, the null space of $I-P$ is on $\overline{[\mathrm{TS}] \mathbb{H}}$, hence on $(I-P) \overline{[\mathrm{TS}] \mathbb{H}}=0$ equivalently, $(I-P)[\mathrm{TS}] \mathbb{H}=0$. Applying the properties of the adjoint to the bounded self-adjoint operators $S, T$ and $(I-P)$ and taking an element $y \in \mathbb{H}$, we have

$$
\begin{aligned}
0 & =\langle y,(I-P)[T S] v\rangle \\
& =\left\langle(I-P)^{*} y,[T S] v\right\rangle \\
& =\langle(I-P) y,(T S-S T) v\rangle \\
& =\left\langle(T S-S T)^{*}(I-P) y, v\right\rangle \\
& =\left\langle\left((T S)^{*}-(S T)^{*}\right)(I-P) y, v\right\rangle \\
& =\left\langle\left(S^{*} T^{*}-T^{*} S^{*}\right)(I-P) y, v\right\rangle \\
& =\langle(S T-T S)(I-P) y, v\rangle \\
& =\langle S T(I-P) y-T S(I-P) y, v\rangle
\end{aligned}
$$

From the choice of $v$, we have $v \neq 0$ hence, we have $S T(I-P) y-T S(I-P) y=0$ implying that $S T(I-$ $P) y=T S(I-P) y=0$.

Let us define $x:=S T(I-P) y=T S(I-P) y$. Then $x \in \mathcal{C}$.

From the relations, $x=S T(I-P) y$ and $x=T S(I-P) y$, since $T=(A+\alpha)^{-1}$ and $S=(B+\alpha)^{-1}$ are invertible, we have 
Likewise, we have

$$
\begin{aligned}
(S T)^{-1} x & =(I-P) y \\
T^{-1} S^{-1} x & =(I-P) y \\
(A+\alpha)(B+\alpha) x & =(I-P) y \\
E F x & =(I-P) y
\end{aligned}
$$

$$
\begin{aligned}
(T S)^{-1} x & =(I-P) y \\
S^{-1} T^{-1} x & =(I-P) y \\
(B+\alpha)(A+\alpha) x & =(I-P) y \\
F E x & =(I-P) y
\end{aligned}
$$

Thus, $x \in D(E F)$ and $x \in D(F E)$ implying that $x \in D(E F) \cap D(F E)$. Therefore, $\mathcal{C} \subseteq D(E F) \cap D(F E)$. The choice of $y$ determining the definition of $x$ was arbitrary, hence, the inclusion $\mathcal{C} \subseteq D(E F) \cap D(F E)$ is true for all $x \in \mathcal{C}$.

(2)

We first show that $(A+\alpha)^{-1} \mathcal{C}=T \mathcal{C}$ is dense in $\mathbb{H}$. If we take $v \perp T \mathcal{C}$ for any $v \in \mathbb{H}$, we only need to show that $v=0$. From $(1)$ above $\mathcal{C}=(A+\alpha)^{-1}(B+\alpha)^{-1}(I-P) \mathbb{H}=T S(I-P) \mathbb{H}$ By choice of $v$, for any $y \in \mathbb{H}$, we have $\langle v, E T S(I-P) y\rangle=0$. Since $E$ and $T$ are inverses of one another, we have

$$
\begin{aligned}
0 & =\langle v, \operatorname{ETS}(I-P) y\rangle \\
& =\langle v, S(I-P) y\rangle \\
& =\left\langle S^{*} v,(I-P) y\right\rangle \\
& =\langle S v,(I-P) y\rangle
\end{aligned}
$$

Thus, $S v \perp(I-P) y$ implying that $S v \in \operatorname{Ran}(P)$. By hypothesis, $P \mathbb{H} \cap S \mathbb{H}=\{0\}$, hence $S v=0$ implying that $v=0$ as required.

We now show that $\mathcal{C}$ is the core of $D(E)$, that is, $\overline{\mathcal{C}}=D(E)$. For any convergent sequence $\left\{x_{n}\right\}_{n=1}^{\infty}$ such that $x_{n} \in \mathcal{C}$ and $x_{n} \rightarrow x$. We expect $x \in D(E)$. Let $x \in D(E)$, since $F \mathcal{C}$ is dense in $\mathbb{H}$ and $D(E) \subseteq \mathbb{H} E x_{n} \rightarrow E x$. Pre-multiplying by the bounded operator $T$, we get $T E x_{n}=x_{n}$ hence

Thus, $x_{n} \rightarrow x$ as required.

$$
x_{n}=T E x_{n} \rightarrow T E x=x .
$$

We do the same for $F$. We show that $(B+\alpha)^{-1} \mathcal{C}=S \mathcal{C}$ is dense in $\mathbb{H}$. If we take $v \perp S \mathcal{C}$ for any $v \in \mathbb{H}$, we only need to show that $v=0$.

From (1) above $\mathcal{C}=(A+\alpha)^{-1}(B+\alpha)^{-1}(I-P) \mathbb{H}=T S(I-P) \mathbb{H}$ equivalently, $\mathcal{C}=S T(I-P) \mathbb{H}$. For any $y \in$ $\mathbb{H}$, we have $\langle v, F S T(I-P) y\rangle=0$. Since $F=S^{-1}$, we have

$$
\begin{aligned}
0 & =\langle v, F S T(I-P) y\rangle \\
& =\langle v, T(I-P) y\rangle \\
& =\left\langle T^{*} v,(I-P) y\right\rangle \\
& =\langle T v,(I-P) y\rangle
\end{aligned}
$$

Thus, $T v \perp(I-P) y$ implying that $T v \in \operatorname{Ran}(P)$. By hypothesis, $P \mathbb{H} \cap T \mathbb{H}=\{0\}$, hence $T v=0$ implying that $v=0$ as required.

We now show that $\overline{\mathcal{C}}=D(F)$. For any convergent sequence $\left\{x_{n}\right\}_{n=1}^{\infty}$ such that $x_{n} \in \mathcal{C}$ and $x_{n} \rightarrow x \in D(F)$. Let $x \in D(F)$, since $F \mathcal{C}$ is dense in $\mathbb{H}$ and $D(F) \subseteq \mathbb{H} F x_{n} \rightarrow F x$. Pre-multiplying by $S$, we get

Thus, $x_{n} \rightarrow x$ as required.

$$
x_{n}=S F x_{n} \rightarrow S F x=x \text {. }
$$

(3)

Let $y \in \mathbb{H}$, then by the preceeding statements, $u=T S(I-P) y \in \mathcal{C}$ hence

$$
E u=E T S(I-P) y=S(I-P) y \in D(T S(I-P))=\mathcal{C}
$$

Hence $E u \in \mathcal{C}$. The set $\mathcal{C}$ is an invariant subpace under $E$.

On the other hand, let $y \in \mathbb{H}$, then by the preceding statements, $u=S T(I-P) y \in \mathcal{C}$ hence

$$
F u=F S T(I-P) y=T(I-P) y \in D(S T(I-P))=\{C\}
$$

Hence $F u \in \mathcal{C}$. The set $\mathcal{C}$ is an invariant subpace under $F$.

So far, we have now established that the two operators $E=A+\alpha$ and $F=B+\alpha$ specially defined above commute pointwise on a common core $\mathcal{C}$ defined above. We now prove that they commute strongly. For easy reference, we denote the collection of operators satisfying the conditions and results of the proposition $\mathbf{3 . 1}$ by $\mathcal{C}_{p s}$. Therefore, $E, F \in \mathcal{C}_{p s}$.

\section{Proposition 3.12}

Let $E=A+\alpha$ and $F=B+\alpha$ be unbounded positive Self-adjoint operators having compact Self-adjoint inverses on a seperable Hilbert Space $\mathbb{H}$ for sufficiently large $\alpha \in \mathbb{R}$. If $E, F \in \mathcal{C}_{p s}$ then $E$ and $F$ commute strongly. 


\section{Proof}

Consider the core $\operatorname{CTS}(I-P)$ as defined in proposition 3.1 where $P$ is a projection on the closure of $[T, S] \mathbb{H}$. For $y \in \mathbb{H}$, we have $x=T S(I-P) y \in \mathcal{C}$.

Since $\mathbb{H}$ is a seperable Hilbert space, we have a represention of $\mathbb{H}$ as

$$
\mathbb{H}=\bigoplus_{i \in \mathbb{N}} \mathbb{H}_{i}
$$

The fact that our operators are Self-adjoint, the subspaces $\mathbb{H}_{i}$ is almost one dimensional.

Let $\mathbb{H}_{i}$ be a non-zero subspace of $\mathbb{H}$ such that there are eigenvalues $\left.\lambda_{E i} \in \sigma(E)\right|_{\mathbb{H}_{i}}$ and $\left.\lambda_{F i} \in \sigma(F)\right|_{\mathbb{H}_{i}}$ for which $P\left(\lambda_{E i}\right)$ and $P\left(\lambda_{F i}\right)$ are spectral measures on $\mathbb{H}_{i}$. Since $E=A+\alpha$ and $F=B+\alpha$ are unbounded positive Selfadjoint operators, we have unique spectral measures $P\left(\lambda_{E i}\right)$ and $P\left(\lambda_{F i}\right)$ where

$$
\begin{aligned}
& A+\alpha=\int_{\mathfrak{J}}\left(\lambda_{E i}+\alpha\right) d P\left(\lambda_{E i}\right) \\
& B+\alpha=\int_{J^{*}}\left(\lambda_{F i}^{*}+\alpha\right) d P\left(\lambda_{F i}^{*}\right)
\end{aligned}
$$

Using the functional calculus for unbounded Self-adjoint operator, we have

$$
(A+\alpha)(B+\alpha)=\int_{J_{\cap J^{*}}}\left(\lambda_{E i}+\alpha\right)\left(\lambda_{F i}^{*}+\alpha\right) d P\left(\lambda_{E i}\right) P\left(\lambda_{F i}^{*}\right)
$$

Let $Q$ be a projection on $\mathbb{H}_{i}$ such that for $T S(I-P) y \in \mathcal{C}$ we have $Q T S(I-P) y \in \mathbb{H}_{i}$ and $\|Q T S(I-P) y\|=1$; then any vector in $\mathbb{H}_{i}$ will be a linear combination of $Q T S(I-P) y$. As such, there exist unique constants $k_{1}$ and $k_{2}$ such that

$$
\begin{aligned}
& P\left(\lambda_{E i}\right) T S(I-P) y=k_{1} T S(I-P) y \\
& P\left(\lambda_{F i}^{*}\right) T S(I-P) y=k_{2} T S(I-P) y
\end{aligned}
$$

This implies that $P\left(\lambda_{F i}^{*}\right) T S(I-P) y$ and $P\left(\lambda_{E i}\right) T S(I-P) y$ are scalar multiples of one another.

Define the minimum vector

$$
v_{\min }=k Q T S(I-P) y=\min \left\{k_{1} T S(I-P) y, k_{2} T S(I-P) y\right\} .
$$

The product of $P\left(\lambda_{F i}^{*}\right) T S(I-P)$ and $P\left(\lambda_{E i}\right) T S(I-P)$ will therefore be

$$
\begin{aligned}
& P\left(\lambda_{E i}\right) T S(I-P) P\left(\lambda_{E i}\right) T S(I-P) y=v_{\text {min }} \\
& P\left(\lambda_{F i}^{*}\right) T S(I-P) P\left(\lambda_{F i}^{*}\right) T S(I-P) y=v_{\text {min }} .
\end{aligned}
$$

The vector $y$ was arbitrary, so is $T S(I-P) y \in \mathcal{C}$, and the relations in equation 3.3 and 3.4 applies for all elements in $\mathcal{C}$. Since the spectral measures are bounded operators, we have the equality

$$
P\left(\lambda_{E i}^{*}\right) T S(I-P) P\left(\lambda_{F i}\right) T S(I-P)=P\left(\lambda_{F i}^{*}\right) T S(I-P) P\left(\lambda_{E i}\right) T S(I-P) .
$$

Equation 3.5 implies that the spectral measures of the operators $E$ and $F$ commute on a common core $\mathcal{C}$ implying that the two operators commute strongly.

\section{References}

[1]. Berezansky, Y.M., Sheftel, Z.G., Us, G.F., (1996), Functional Analysis, 2,Vysha Shkola: Kiev.

[2]. Gohberg, I., Goldberg, S. \& Kaashoek, A. M. (1990), Classes of Linear Operators Vol 1, Springer Basel AG; Basel, Switzerland.

[3]. Martin, S., (2009), Principals of Functional Analysis, 2nd ed., 36, American Mathematical Society; Rhode Island

[4]. Nelson, E., (1959), Analytic vectors, Annals of Mathematics, 70, 572-614.

[5]. Schmüdgen, K. \& Friedrich, J., (1984), On commuting bounded Self-adjoint operators II. Birkhauser Verlag, Basel. 7, 815-1984.

[6]. Schmüdgen, K., (1986), A Note on Commuting Unbounded Self-adjoint Operators Affiliated to Properly infinite Von Neumann Algebras II. Bulletin of the London Mathematical Society, 18(3),287-292. https://doi.org/10.1112/blms/18.3.287.

[7]. Schmüdgen, K., (1988), Strongly commuting Selfadjoint operators and commutants of unbounded operator algebras. American Mathematical Society, 102(2), 365-372.

[8].Schmüdgen, K., (2012), Unbounded Self-adjoint Operators on Hilbert Space, New York: Springer GTM 265. 